\title{
Localized Malignant Pleural Mesothelioma Showing a Thoracic Mass and Metastasizing to the Stomach
}

\author{
Hidetoshi Hayashi ${ }^{1}$, Kenji Notohara ${ }^{2}$, Hiroshige Yoshioka ${ }^{1}$, Tomoaki Matsuoka ${ }^{3}$, \\ Hiroyoshi Ikeda ${ }^{4}$, Koichi Kagawa ${ }^{5}$, Toshio Fukuoka ${ }^{6}$ and Tadashi Ishida ${ }^{1}$
}

\begin{abstract}
Malignant mesothelioma typically shows either diffuse tumors or multiple pleura-based nodules. Localized malignant mesothelioma is rare. In this case report, a 70-year-old man with left chest wall tumor underwent tumor resection, and the lesion was pathologically diagnosed as biphasic malignant mesothelioma. Tumor recurrence was detected in the stomach due to vomiting of blood, and also spread to the mediastinal lymph node, and bone 3 months postoperatively. Total gastrectomy was performed and the histopathological diagnosis of metastasis of mesothelioma was made. In the previously reported cases, all of the localized malignant mesothelioma arose in the pleural space and there was no metastasis of localized malignant mesothelioma to the stomach. In the present case, gross and histological examinations were performed for both the primary lesion and gastric metastatic tumor. Though it was very difficult to distinguish mesothelioma from sarcoma and other chest wall tumors, immunochemical staining was able to facilitate making the diagnosis. This case suggests that localized malignant mesothelioma is capable of showing multiple forms and a variety of clinical courses. Localized malignant mesothelioma can arise primarily from the chest wall.
\end{abstract}

Key words: localized mesothelioma, thoracic-mediastinum, chest wall

(Inter Med 49: 671-675, 2010)

(DOI: 10.2169/internalmedicine.49.2592)

\section{Introduction}

Malignant mesotheliomas are highly aggressive neoplasms that arise primarily from the surface serosal cells of the pleural, peritoneal, and pericardial cavities. Epidemiologic studies have shown that exposure to asbestos fibers is the primary cause of the disease.

Patients often show either diffuse tumors or multiple serosal membrane-based nodules. The diffuse spread over the serosal surfaces is fundamental to the diagnosis of malignant mesothelioma. However, several case reports of localized malignant mesothelioma have been published. In these reports, all of the tumors were localized to the pleural space without any metastasis to other organs.

In this paper, we report a case of malignant pleural meso- thelioma forming a localized mass in the chest wall. The patient had an aggressive clinical course with distant metastases to multiple organs, including the stomach.

\section{Case Report}

A 70-year-old man noticed a lump in his left chest wall. He had been suffering from chest discomfort for two months. An abnormal shadow in the left lower lung field was detected on chest X-ray (Fig. 1), and CT showed a tumor in the chest wall (Fig. 2) without asbestos exposure, that is asbesto plaque in pleura or fibrotic change in the lung. The patient denied occupational exposure to asbestos, and there were no abnormal physical findings or laboratory examinations (Table 1) except for the hard tumor palpable on the left chest wall. Pathological examination of a biopsy

\footnotetext{
${ }^{1}$ Department of Respiratory Medicine, Kurashiki Central Hospital, Kurashiki, ${ }^{2}$ Department of Pathology, Kurashiki Central Hospital, Kurashiki, ${ }^{3}$ Department of Thoracic Surgery, Kurashiki Central Hospital, Kurashiki, ${ }^{4}$ Department of Surgery, Kurashiki Central Hospital, Kurashiki, ${ }^{5}$ Department of Gastroenterology, Kurashiki Central Hospital, Kurashiki and ${ }^{6}$ Department of General Medicine, Kurashiki Central Hospital, Kurashiki
}

Received for publication June 15, 2009; Accepted for publication December 23, 2009

Correspondence to Dr. Hidetoshi Hayashi, hideT31@gmail.com 


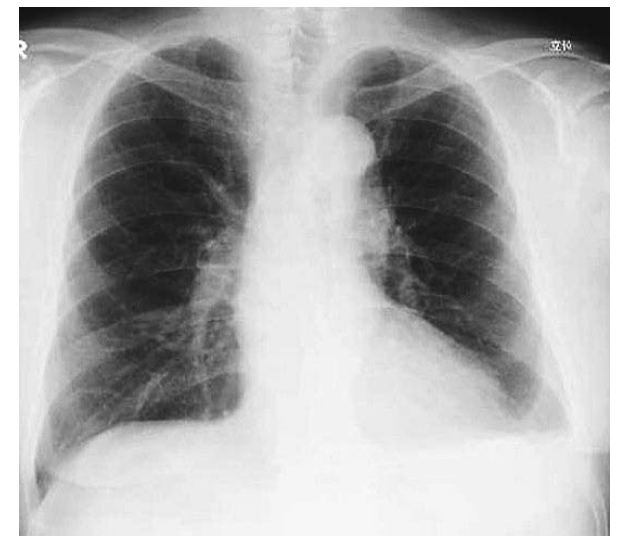

Figure 1. An abnormal shadow in the left lower lung field.

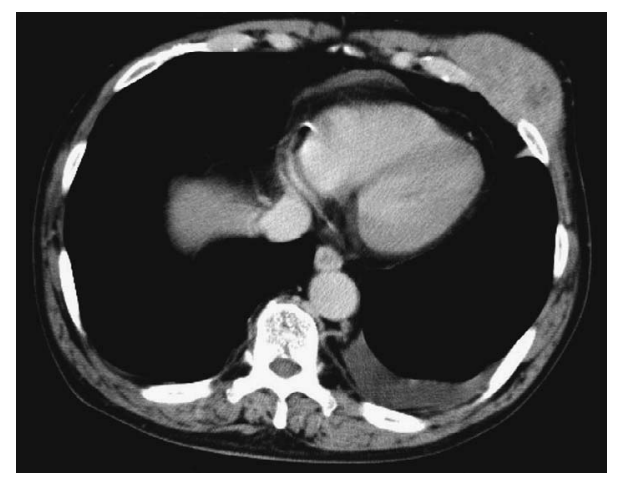

Figure 2. CT showed a tumor in the left chest wall.

was suggestive of high-grade pleomorphic sarcoma, but was not conclusive.

The patient underwent tumor resection, including the left ribs and chest wall. Macroscopically, the resected tumor was solid, and measured $10 \mathrm{~cm}$ in diameter (Fig. 3). The cut surface appeared fish-shape like, and small foci of necrosis were noted. The main portion of the tumor was located in the anterior chest wall, but a small portion of the mass was found to be adjacent to the pleura. Histologically, the tumor consisted of mainly spindle-shaped pleomorphic tumor cells that showed no apparent lines of differentiation (Fig. 4). The presence of necrosis was confirmed histologically.

Lymphoplasmacytic infiltrates were scattered within the tumor. Adjacent to the pleura, there was a small portion with epithelioid tumor cells that formed solid cell nests with an occasional tubulopapillary pattern. These cells had a round nucleus, a small but prominent nucleolus and eosinophilic-abundant cytoplasm. Similar tumor cells were also found focally on the pleural surface. Immunohistochemical stains revealed the expression of EMA, cytokeratin (AE1/AE3), high molecular weight cytokeratin (34ßE12), WT-1, calretinin and podoplanin (D2-40) in the epithelioid portion, although all of these markers were completely negative in the pleomorphic sarcomatoid area. TTF-1 and CEA were entirely negative. Despite the expression of cytokeratin and EMA, synovial sarcoma was ruled out because Bcl-2
Table 1. Laboratory Data on First Visiting

\begin{tabular}{|c|c|c|c|}
\hline \multicolumn{2}{|c|}{ Hematology } & \multicolumn{2}{|c|}{ Biochemistry } \\
\hline WBC & $7300 / \mu \mathrm{L}$ & TP & $7.9 \mathrm{~g} / \mathrm{dL}$ \\
\hline Neu & $74.2 \%$ & Alb & $3.6 \mathrm{~g} / \mathrm{dL}$ \\
\hline Lym & $16.8 \%$ & T-bil & $0.5 \mathrm{mg} / \mathrm{dL}$ \\
\hline Mono & $5.3 \%$ & AST & $37 \mathrm{IU} / \mathrm{L}$ \\
\hline Eos & $2.9 \%$ & ALT & $46 \mathrm{IU} / \mathrm{L}$ \\
\hline Bas & $0.8 \%$ & LDH & $147 \mathrm{IU} / \mathrm{L}$ \\
\hline RBC & $3.45 \times 10^{6} / \mu \mathrm{L}$ & $\gamma$-GTP & $15 \mathrm{IU} / \mathrm{L}$ \\
\hline $\mathrm{Hb}$ & $10.8 \mathrm{~g} / \mathrm{dL}$ & BUN & $12 \mathrm{mg} / \mathrm{dL}$ \\
\hline \multirow[t]{2}{*}{ Plt } & $26.8 \times 10^{4} / \mu \mathrm{L}$ & CRN & $1.09 \mathrm{mg} / \mathrm{dL}$ \\
\hline & & $\mathrm{Na}$ & $140 \mathrm{mEq} / \mathrm{L}$ \\
\hline \multicolumn{2}{|c|}{ Serum tumor marker } & $\mathrm{K}$ & $4.1 \mathrm{mEq} / \mathrm{L}$ \\
\hline CEA & $1.4 \mathrm{ng} / \mathrm{mL}$ & $\mathrm{Cl}$ & $104 \mathrm{mEq} / \mathrm{L}$ \\
\hline $\mathrm{SCC}$ & $1.5 \mathrm{ng} / \mathrm{mL}$ & CRP & $5.21 \mathrm{mg} / \mathrm{dL}$ \\
\hline CYFRA & $<0.1 \mathrm{ng} / \mathrm{mL}$ & & \\
\hline NSE & $5.2 \mathrm{ng} / \mathrm{mL}$ & & \\
\hline Pro-GRP & $37.6 \mathrm{pg} / \mathrm{mL}$ & & \\
\hline
\end{tabular}

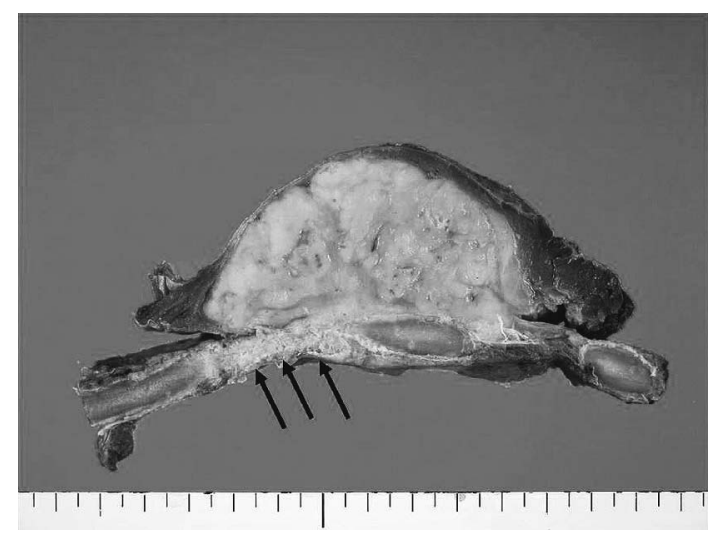

Figure 3. Macroscopically, the resected tumor was solid, the cut surface was pale white with spots of blood, and showed involvement of the rib and parietal pleura.

was negative. Thus, the tumor was diagnosed as a biphasic type of malignant mesothelioma.

Complete en bloc resection was confirmed histologically. The postoperative course was uneventful, and the patient was discharged without complication 7 days after surgery. Three months after the surgery, the patient complained of hematemesis. Radiological examinations depicted possible metastatic lesions in the stomach, mediastinal lymph nodes and bones (spine and breast bone). He received two courses of chemotherapy (cisplatin and pemetrexed), but the tumor size increased. A total gastrectomy was performed to relieve the gastric obstruction (Fig. 5). There was no change in the peritoneal cavity during the gastric tumor operation.

Histologically, the gastric mass resembled the sarcomatoid portion of the thoracic tumor (Fig. 6). Although there were no components that suggested a diagnosis of malignant mesothelioma, immunostaining revealed focal expression of 

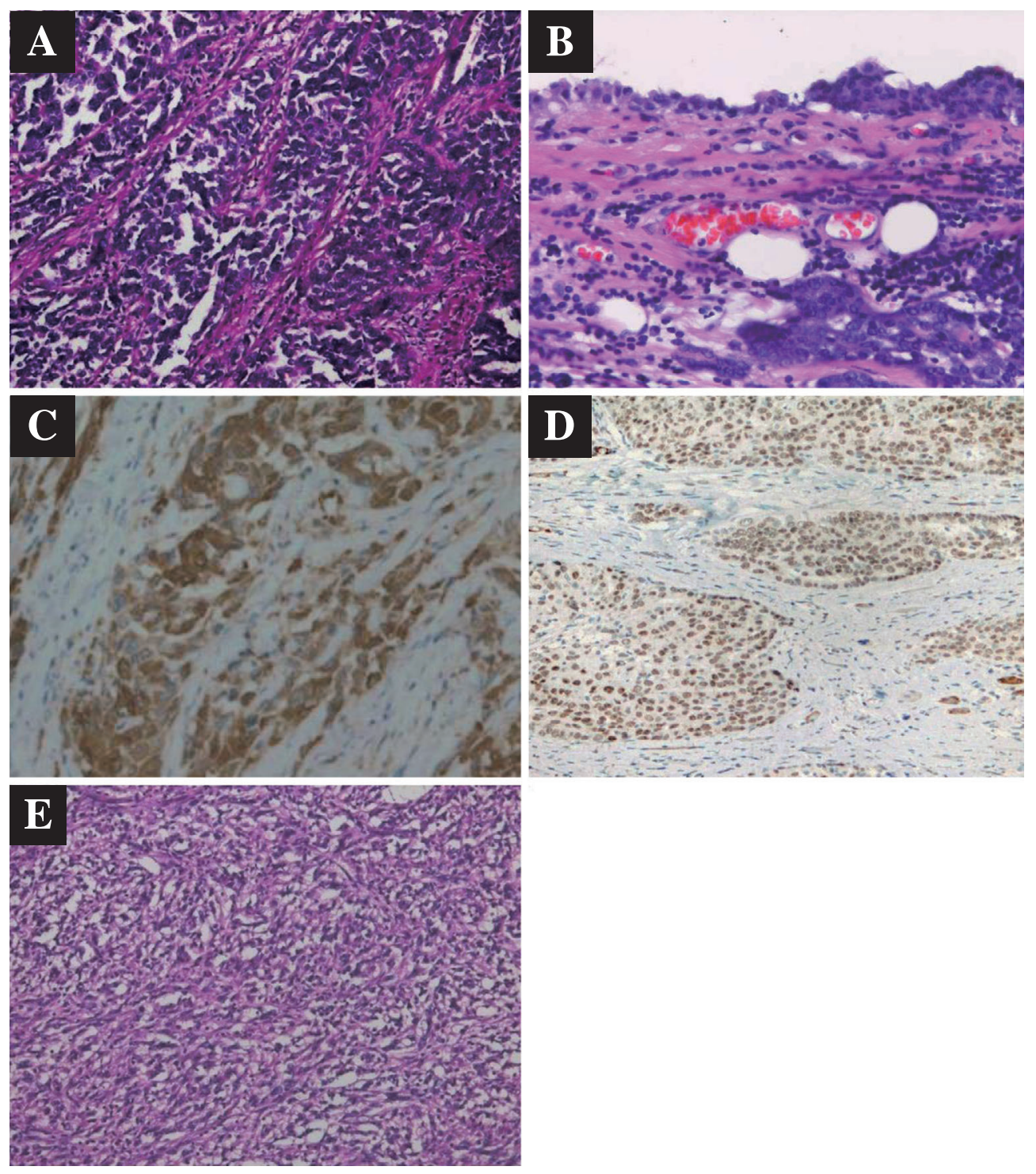

Figure 4. Histological and immunohistochemical features of the chest wall tumor. (A, B, E: Hematoxylin and Eosin staining, C: Calretinin staining, D:WT-1 staining). A: Epithelium component. B: The tumor cells were also found focally on the serosal surface. C, D: The epithelial component was positive for calretinin and WT-1. E: Sarcomatoid component.

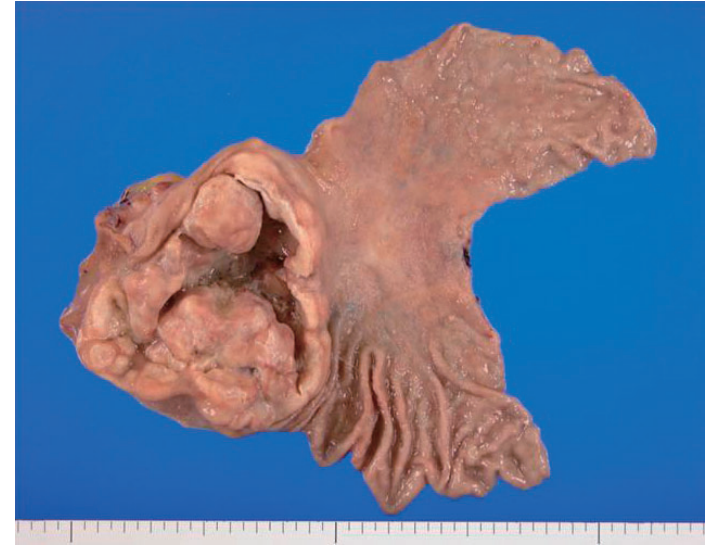

Figure 5. The gastric metastasis was a single bulky mass that resembled a primary gastric tumor.
EMA, smooth muscle actin, WT-1, calretinin and podoplanin. Markers for various sarcomas, such as desmin, c-kit, CD31, CD34 and S-100 protein were all negative. A diagnosis of metastatic malignant mesothelioma was rendered. The patient received palliative care after the second operation. His condition deteriorated quickly and he died 8 months after the initial operation.

\section{Discussion}

Malignant mesothelioma is usually associated with occupational asbestos exposure and usually shows either diffuse tumors or multiple pleura-based nodules (1). However, rarely, mesotheliomas manifest as a localized mass, and these are designated as localized malignant mesothelioma. The localized malignant mesothelioma was first described 

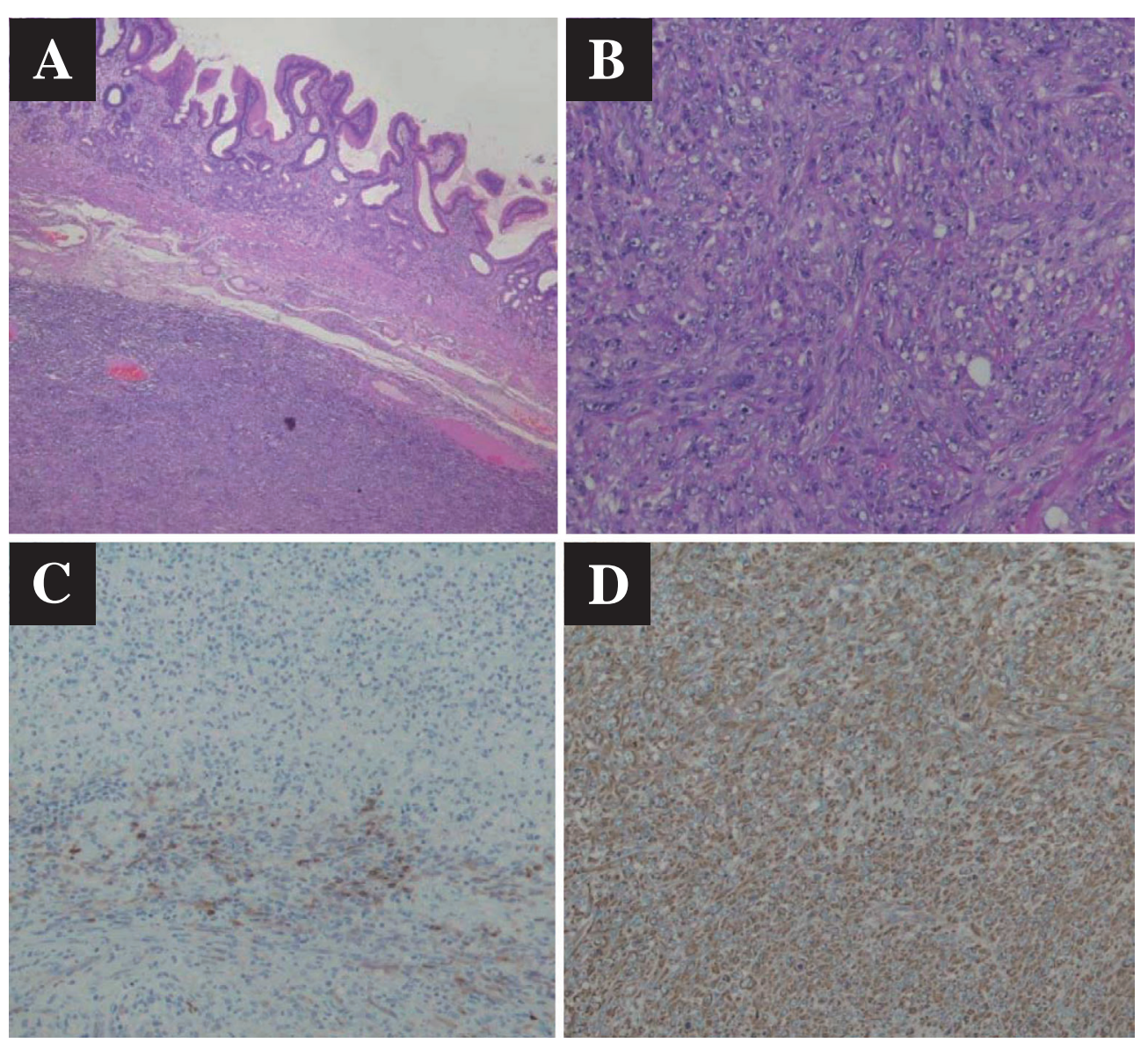

Figure 6. Metastatic tumor mainly showed sarcomatoid features including some epithelioid components (A, B: Hematoxylin and Eosin staining, C: Calretinin staining, D: Vimentin staining).

by Crotty et al in 1994 (2) and several cases have been reported since then (3-12). Allen et al proposed the diagnostic criteria for localized malignant mesothelioma (3). These criteria are: (i) radiological, surgical, or pathological evidence of a localized serosal/subserosal (but not organ-centered) tumor mass without evidence of diffuse serosal spread; and (ii) a microscopic pattern identical to that found in ordinary diffuse malignant mesothelioma. Localized malignant mesothelioma should be distinguished from the conventional diffuse type because of the different biological behaviors and localized presentations.

The tumor presented here is an example of localized malignant mesothelioma. In addition, this case had three unique clinicopathological features. First, the tumor was located mainly in the anterior chest wall. The radiological diagnosis was a chest wall tumor because the pleural involvement of the tumor was too small to be depicted radiologically. The histological differential diagnoses of chest wall tumors with sarcomatoid features like this case are diverse, including primary bone and soft tissue neoplasms, hematological malignancies, local invasion of pulmonary or pleural neoplasms and metastatic lesions. In addition, contrary to metastatic chest wall neoplasms or local invasion, primary neoplasms are rare, representing only 5\% of whole chest wall neoplasms $(13,14)$, which makes the diagnosis of chest wall neoplasms more challenging. In fact, we could not render a specific diagnosis with a biopsy tissue. However, the resected specimen was diagnosed as localized malignant mesothelioma because the epithelioid component that was histologically compatible with malignant mesothelioma was identified adjacent to the pleura, and even on the pleural surface, and immunohistochemically it expressed cytokeratin, calretinin and podoplanin. Markers for carcinomas, such as CEA and TTF-1, were negative. Synovial sarcoma was a differential diagnosis but it was unlikely because Bcl-2 was negative. Our literature search did not find a report of localized malignant mesothelioma in the chest wall.

Second, the sarcomatoid portion of the tumor was negative for cytokeratin. In most cases, sarcomatoid mesotheliomas are positive, at least focally, for cytokeratin, and, according to a recent monograph, the diagnosis of cytokeratinnegative sarcomatoid mesothelioma should be limited to those with typical gross distribution and no prior history of soft tissue sarcomas (15). We propose that connection to the typical epithelioid mesothelioma, as seen in the present case, is another strong indicator for the diagnosis of sarcomatoid mesothelioma, and it is advisable for such cases to be examined with multiple blocks for the diagnostic features.Third, metastatic lesions developed in multiple organs, and the patient died in a short period of time. The clinical course is 
unusual for mesotheliomas, and rather resembles high grade carcinomas and sarcomas. The gastric metastasis was a single bulky mass that resembled a primary gastric tumor, and was different from the wide spread serosal involvement that is commonly seen in mesotheliomas. In the largest autopsy series of patients with MPM, 54\% to $82 \%$ had distant metastases, with the most frequently involved organs being the liver, adrenal gland, kidney, and contralateral lung (16). King et al reported 22 cases of metastasis from malignant mesothelioma (17). They reported widespread metastases and direct tumor extension. In one case, the tumor involved the mucosal surface of the gastrointestinal tract, but there was no stomach metastasis. In the present case, there was no evidence of abnormality in the peritoneal cavity during the gastric tumor operation, denying a possibility of peritoneal mesothelioma and chest wall metastasis.

In summary, we were able to diagnose localized mesothelioma in the chest wall that metastasized to multiple organs. We considered this case important because localized malignant mesothelioma may resemble extrathoracic tumors and show an aggressive clinical course. We should consider localized malignant mesothelioma as a diagnosis for cases with chest wall tumors.

\section{Acknowledgement}

We are grateful to Dr. Kouki Inai, Department of Pathology, Graduate School of Biomedical Sciences, Hiroshima University, Hiroshima, Japan for his expert opinion on the pathological diagnosis.

\section{References}

1. Attanoos RL, Gibbs AR. Pathology of malignant mesothelioma. Histopathology 30: 403-418, 1997.

2. Crotty BT, Myers JL, Katzenstein AA, Tazelaar HD, Swensen SJ, Churg A. Localized malignant mesothelioma. AM J Surg Pathol 18: 357-363, 1994.

3. Allen TC, Cagle PT, Churg AM, et al. Localized malignant mesothelioma. Am J Surg Pathol 29: 866-873, 2005.

4. Erkilic S, Sari I, Tuncozgur B. Localized pleural malignant mesothelioma. Pathol Int 51: 812-815, 2001.

5. Gomez-Roman JJ, Mons-Lera R, Olmedo IS, Val-Bernal JF. Flow cytometric analysis of a localized malignant mesothelioma. Am J Surg Pathol 73: 1292-1294, 2002.

6. Imura J, Ichikawa $\mathrm{K}$, Takeda J, et al. Localized malignant mesothelioma of the epithelial type occurring as a primary hepatic neoplasm: a case report with review of the literature. APMIS 110: 789-794, 2002.

7. Matsukuma S, Aida S, Hata Y, Sugiura Y, Tamai S. Localized malignant peritoneal mesothelioma containing rhabdoid cells. Pathol Int 46: 389-391, 1996.

8. Ojeda HF, Mech K, Hicken W. Localized malignant mesothelioma: a case report. Am Surg 64: 881-885, 1998.

9. Okamura H, Kamei T, Mituno A, Hongo H, Sakuma N, Ishihara T. Localized malignant mesothelioma of the pleura. Pathol Int 51: 654-660, 2001.
10. Shimazaki H, Aida S, Iizuka Y, Yoshizu H, Tamai S. Vacuolated cell mesothelioma of the pericardium resembling liposarcoma: a case report. Hum Pathol 31: 767-770, 2001.

11. Umezu H, Kuwata K, Ebe $Y$, et al. Microcystic variant of localized malignant mesothelioma accompanying an adenomatoid tumor-like lesion. Pathol Int 52: 416-422, 2002.

12. Val-Bernal JF, Figols J, Gomez-Romain JJ. Incidental (solitary) epithelial mesothelioma of the pericardium: case report and literature review. Cardiovasc Pathol 11: 181-185, 2002.

13. Incarbone M, Pastorino U. Surgical treatment of chest wall tumors. World J Surg 25: 218-230, 2001.

14. Hsu PK, Hsu HS, Lee HC, et al. Management of primary chest wall tumors: 14 years' clinical experience. J Clin Med Assoc 69: 377-382, 2006.

15. Churg A, Cagle PT, Roggli VI. Tumors of the serosal membranes. In: AFIP Atlas of Tumor Pathology, 4th series, fascicle 3. ARP Press, Washington, DC, 2006: 33-82.

16. Law MR, Hodson ME, Heard BE. Malignant mesothelioma of the pleura: relation between histological type and clinical behavior. Thorax 37: 810, 1982.

17. King JA, Tucker JA, Wong SW. Mesothelioma: A study of 22 cases. South Med J 90: 199-205, 1997.

(C) 2010 The Japanese Society of Internal Medicine http://www.naika.or.jp/imindex.html 